\title{
Invasive Weed Optimization (IWO) Algorithm for Control of Nulls and Sidelobes in a Concentric Circular Antenna Array (CCAA)
}

\author{
Thotakura T. \\ Ramakrishna Satish Raj \\ M.TECH Student, \\ Dept. of E.C.E, \\ S.R.K.R Engineering College, \\ Affiliated to Andhra University, \\ Andhra Pradesh, India.
}

\author{
P. Krishna Kanth Varma \\ Assistant Professor, \\ Dept. of E.C.E, \\ S.R.K.R Engineering College, \\ Affiliated to Andhra University, \\ Andhra Pradesh, India.
}

\author{
P.V. Rama Raju, PhD \\ Professor, \\ Dept. of E.C.E, \\ S.R.K.R Engineering College, \\ Affiliated to Andhra University, \\ Andhra Pradesh, India.
}

\begin{abstract}
The ever increasing wireless communication research is giving rise to a plethora of new communication techniques. There is also a wide spread increase in the use of existing systems such as mobiles, Wi-Fi, RADARS, Space communications etc. Consequently, the electromagnetic spectrum is getting overcrowded and there is an increase in interference due to use of multiple devices with in a small cluster. So, design of antennas with small sidelobe levels and nulls in interference direction is needed. This paper illustrates the procedure for placing nulls in the interference direction while reducing sidelobe level (SLL) of a CCAA under the constraint of fixed FNBW (as in the case of uniform excitation). IWO is used to find the excitation amplitudes of a three ring CCAA that satisfy the desired goal better. Three design examples are provided. IWO is more robust and efficient algorithm than GA, PSO and Simulated Annealing etc.
\end{abstract}

\section{General Terms}

Antenna Array Optimization, Global Optimization Algorithms

\section{Keywords}

Invasive Weed Optimization (IWO) algorithm, Concentric Circular Antenna Array (CCAA), Nulls, Sidelobe levels (SLL), First Null Beam Width (FNBW), Real Coded Genetic Algorithm (RCGA).

\section{INTRODUCTION}

Wireless Communication has become an indispensable part of our society. The variety of existing or future wireless communication networks is growing and includes digital cellular networks, wireless sensor networks, wireless networking for internet access, short-range point-to-point wireless connectivity, mobile broadcasting systems, global navigation satellite systems, body-centric wireless communication systems and surveillance systems [1]. The development of these different wireless systems has become possible only with the development of different radiating structures called 'antennas', with different radiating characteristics. The stringent requirements imposed by these communication networks proved challenging to design a single antenna that could provide the desired radiation pattern. So, a group of antennas called 'antenna array' is used as a single entity to meet the challenges.
An 'antenna array' is an assembly of radiating elements in an electrical and geometrical configuration. In most cases, the elements are identical. The total field of the antenna array is found by the vector addition of the fields radiated by each individual element. An antenna array can be used to provide highly directive patterns, to increase the overall gain, to cancel out interference and to steer the beam in a particular direction. There are at least five controls that can be used to shape the overall pattern of the antenna array [2]. They are:

- The geometrical configuration of the overall array (linear, circular, rectangular, spherical, etc.).

- The relative displacement between the elements.

- The excitation amplitudes of the individual elements.

- The excitation phase of the individual elements.

- The relative pattern of individual elements

By varying these control parameters, coverage areas with different radiation patterns can be generated.

The design of antenna array using classical analytical techniques is quite complicated and cumbersome because of the large number of parameters and the difficulty in calculating performance characteristics. So much of the present research is being focused on using evolutionary optimization algorithms to solve electromagnetic problems related to antenna arrays.

There are many evolutionary algorithms such as Genetic Algorithm (GA), Particle Swarm Optimization (PSO), Ant Colony Optimization (ACO), Simulated Annealing and Invasive Weed Optimization (IWO). Of these Invasive Weed Optimization is a promising technique for designing antenna arrays. IWO is found to be more robust and efficient method.

In this paper, the use of Invasive Weed Optimization in controlling nulls and sidelobe level of a three-ring CCAA is presented. The first null beam width is maintained constant. Excitation amplitudes of CCAA are manipulated to achieve the desired goal.

The rest of the sections are arranged as follows: Section 2 contains design equations of the CCAA. In section 3, IWO is introduced and its features are explained. Section 4 presents results and finally section 5 ends with the conclusion. 


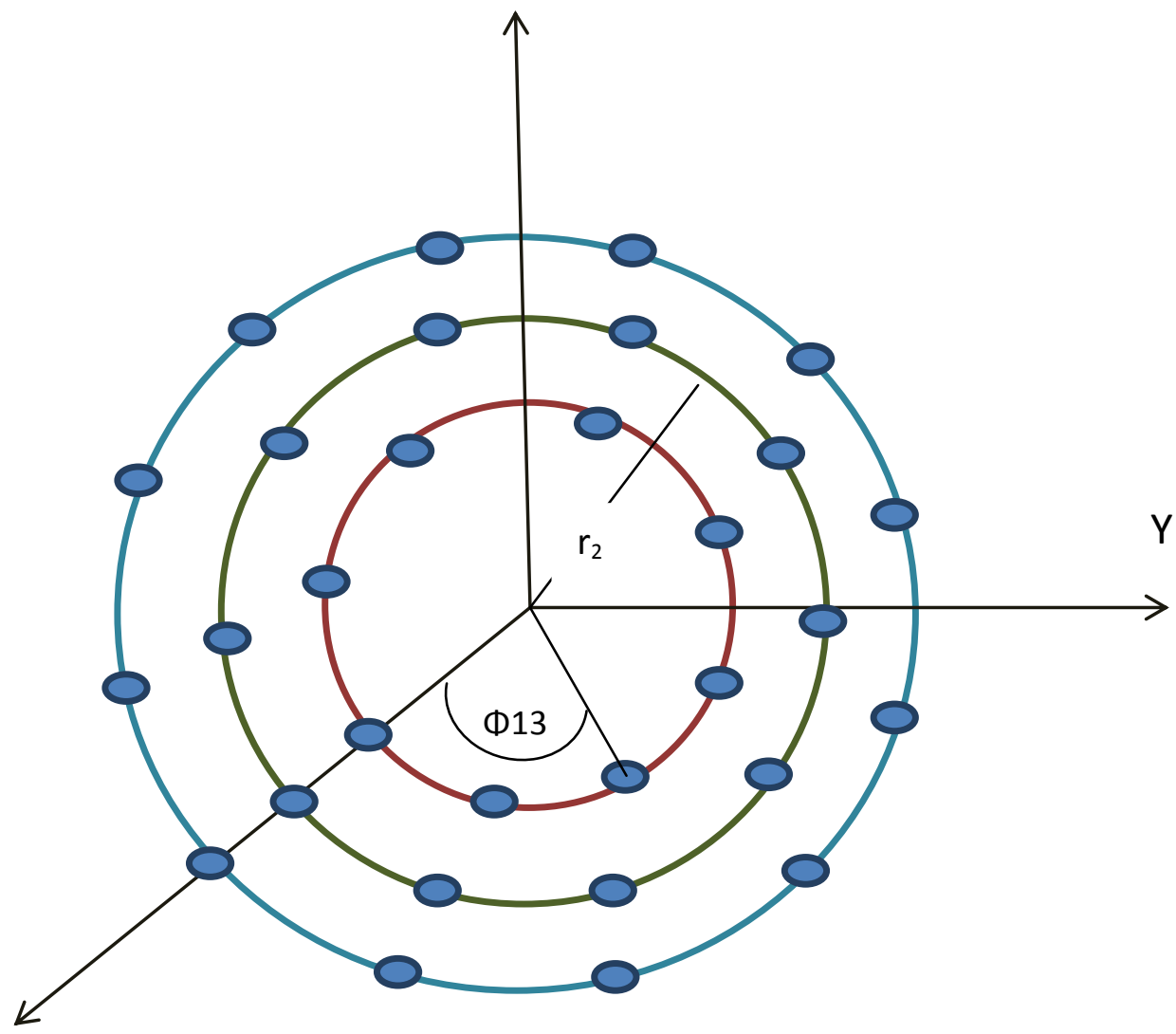

$X$

Fig 1: A 3-Ring Concentric Circular Antenna Array with 8, 10 and 12 elements

\section{DESIGN EQUATIONS}

A concentric Circular Antenna Array contains different elements arranged in a number of concentric circles with varying radii. CCAA is widely used in RADARs, mobile communications, space communications etc. CCAA are more versatile and can provide more symmetric patterns with lower sidelobe levels. CCAA has the advantage of all azimuthal scan capability $[2,3]$.

A CCAA with three rings is shown in Fig. 1 . The $\mathrm{m}^{\text {th }}(\mathrm{m}=1$, $2 \ldots \mathrm{M})$ ring has a radius of $r_{m}$ and contains $N_{m}$ elements. Assuming the elements are isotropic, the array factor can be written as shown in equation $1[3,4]$.

$A F(\phi, I)=\sum_{m=1}^{M} \sum_{n=1}^{N_{m}} I_{m n} \exp \left[j\left(K r_{m} \sin \theta \cos \left(\phi-\phi_{m n}\right)+\alpha_{m n}\right)\right](1)$

Where, $I_{m n}$ denotes the excitation current amplitude of the $n^{\text {th }}$ element of the $m^{\text {th }}$ ring. $K=2 \pi / \lambda$ is the wavenumber and $\lambda$ is the wavelength. $\Theta$ and $\varnothing$ denote the elevation and azimuth angle measured from positive $\mathrm{Z}$ and $\mathrm{X}$ axes respectively. Assuming the elevation angle $\Theta=90^{\circ}$, the array factor can be written as a function of $\varnothing$.
The angle $\emptyset_{\mathrm{mn}}$ is the angular position of the $\mathrm{n}^{\text {th }}$ element of the $\mathrm{m}^{\text {th }}$ ring measured from positive $\mathrm{X}$-axis. Assuming uniform spacing between the elements, it is given as

$\phi_{m n}=2 \pi\left(\frac{n-1}{N_{m}}\right) ; m=1, . ., M, n=1, . ., N_{m}(2)$

The term $\alpha_{\mathrm{mn}}$ is the residual phase which is a function of angular position $\varnothing_{\mathrm{mn}}$ and ring radius $\mathrm{r}_{\mathrm{m}}$

$\alpha_{m n}=-K r_{m} \cos \left(\phi_{0}-\phi_{m n}\right) ; m=1, . ., M, n=1, . ., N_{m}$

Where $\emptyset^{0}$ is the desired value of $\varnothing$ towards which main lobe peak is to be directed.

\subsection{Cost Function}

In optimization algorithms, the 'Cost function' also called 'Objective function' is formulated to find the control parameter values that satisfy the desired goal better. Usually the control parameters need to be selected within certain constrained limits. Here in this paper, the Cost function is designed to achieve the desired goal of introducing nulls and reducing sidelobe level while maintaining fixed FNBW [4]. The Cost Function, $\mathrm{CF}$ is given as 


$$
\begin{gathered}
C F=\frac{C_{1} * \frac{\mid \pi_{i=1}^{m} A F\left(\text { null }_{i}\right) \mid}{\left|A F_{\text {max }}\right|}+C_{2} *\left(S L L_{c u r}-S L L_{d e s}\right)}{+C_{3} * \mid\left(F N B W_{c o m}-F N B W_{\left(I_{m n}=1\right)} \mid\right.}
\end{gathered}
$$

In first term, $m$ is the maximum number of positions where the null can be imposed. In this paper, the value of ' $\mathrm{m}$ ' is considered to be two. AF (nulli) is the value of array factor at the particular null position and $\mathrm{AF}_{\text {max }}$ is the maximum value of array factor.

The second term is used to reduce SLL. SLL $\mathrm{Sur}_{\text {is }}$ is thevel of the sidelobe with the highest peak in $\mathrm{dB}$ for the current iteration and $\mathrm{SLL}_{\mathrm{des}}$ is the desired sidelobe level in $\mathrm{dB}$.

The third term in equation 4 is used to maintain constant FNBW as in the case of uniform excitation. $\mathrm{FNBW}_{\text {com }}$ is the first null beam width for the current iteration and FNBW $\left(\mathrm{I}_{\mathrm{mn}}\right.$ $=1$ ) is the FNBW for the uniform excitation case.

$\mathrm{C}_{1}, \mathrm{C}_{2}, \mathrm{C}_{3}$ are the weighting coefficients used to control the significance of each term. Since main aim is to introduce nulls, $\mathrm{C}_{1}$ must be greater than $\mathrm{C}_{2}$ and $\mathrm{C}_{3}$. In this paper they are taken as 18,2 and 1 respectively.

\section{IWO AND ITS FEATURES \\ 3.1 IWO}

Invasive Weed Optimization algorithm was first introduced by Mehrabian and Lucus in 2006. It was inspired from colonization of weeds. The behavior of invasive weeds in a cropping system is as follows [5]:

Weeds invade the fields by dispersing their seeds with the help of air. These seeds occupy the available spaces and grow into flowering weeds by utilizing the available resources. Those weeds that adapt well to the environment produce more seeds than others. These new weeds are dispersed randomly in the field and they grow into flowering weeds and the process continues. This competition among the weeds makes them adapt well to the environment.

The IWO is explained with the help of a flowchart in figure 2.

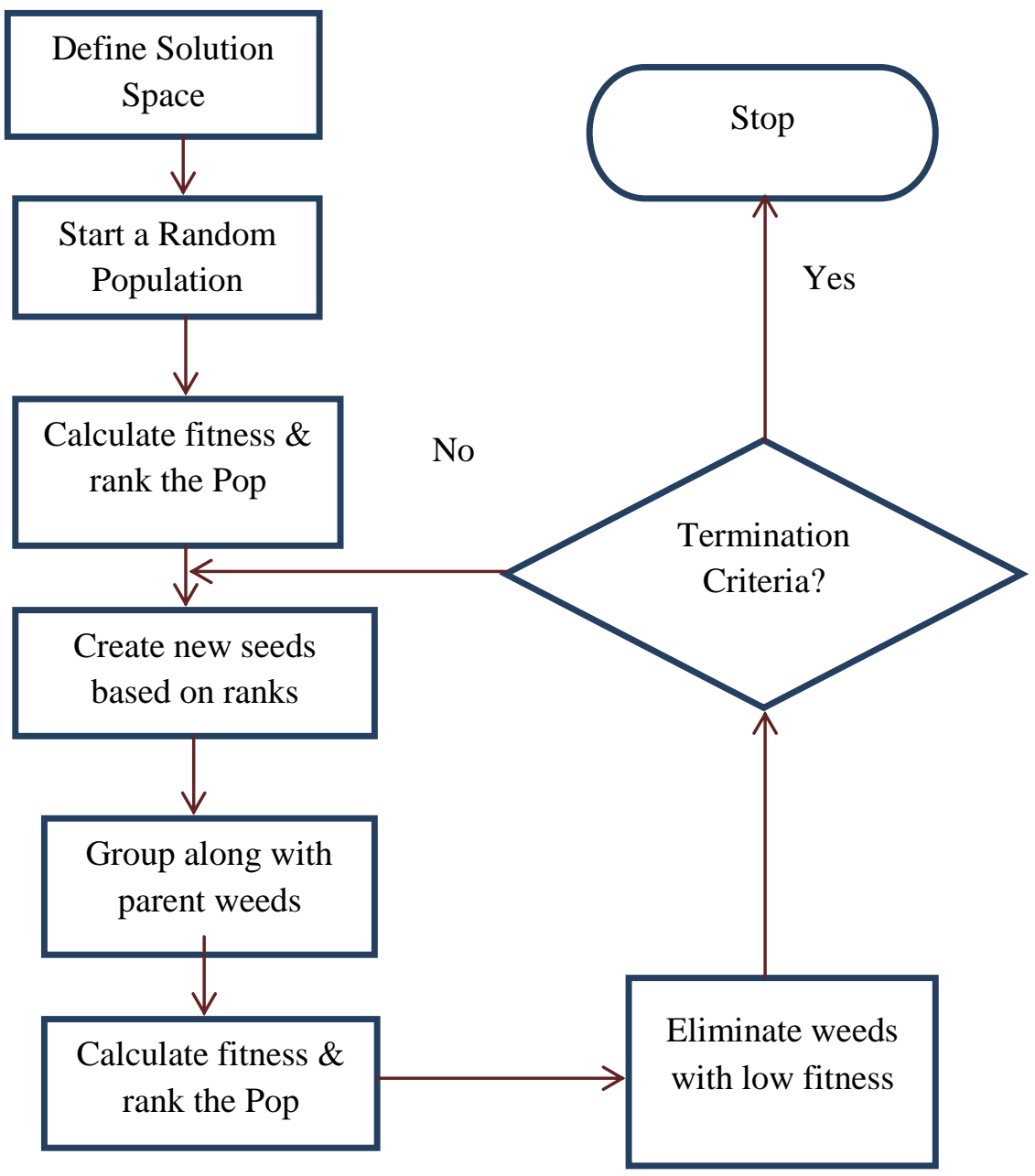

Fig 2: Flowchart of IWO 


\subsection{Algorithm}

The IWO algorithm is explained as follows

$>$ First define the solution space (limits) for each variable of the seed.

$>$ Generate a random population of seeds with each variable of the seed generated within the prescribed limits.

$>$ Evaluate fitness of each seed and rank them based on fitness. The seeds should now be called flowering weeds.

$>$ The flowering weeds are now allowed to produce new seeds based on their rank. The number of seeds produced by a weed ranges between $S_{\min }$ and $S_{\max }$ linearly increasing from the lowest ranked weed to the highest ranked weed.

$>$ The seeds are generated using the normally distributed random numbers with mean equal to the location of the producing weeds and varying standard deviations. The standard deviation at the current iteration is given as

$$
\sigma_{\text {iter }}=\frac{\left(\text { iter }_{\max }-\text { iter }\right)^{n}}{\left(\text { iter }_{\max }\right)^{n}}\left(\sigma_{\text {ini }}-\sigma_{\text {fin }}\right)+\sigma_{\text {fin }}(5)
$$

Here iter $_{\max }$ is the maximum number of iterations. $\sigma_{\text {ini }}$ and $\sigma_{\text {fin }}$ are defined initial and final standard deviations, respectively and $\mathrm{n}$ is the nonlinear modulation index.

$>$ The fitness of the newly generated seeds is assessed and they become the flowering weeds. Now, they are ranked together with their parents based on fitness.

$>$ The weeds with lower fitness are eliminated to reach the maximum allowed weeds in the colony, $\mathrm{P}_{\max }$.

$>$ Survived weeds can produce new seeds based on their rank. This process continues until the termination criterion is met. The termination criterion is usually taken as the no of iterations or certain cutoff fitness value etc.

\subsection{Features}

The important features of IWO are [6]

$>$ Competitive Exclusion: One important property of the IWO is that it allows all of the agents to participate in the reproduction process. Plants with more fitness produce more seeds than less fit plants, which tends to improve the convergence of the algorithm.

$>$ Seeds with different sizes: IWO allows the weeds to reproduce without mating. So, each seed may have different number of variables during the optimization process.

\section{EXPERIMENTAL RESULTS}

The IWO algorithm proposed in the previous section is implemented and simulated using Matlab 2013a for a CCAA having 3 circular rings with radii of $0.5917 \lambda, 0.6944 \lambda$, $0.8463 \lambda$ respectively. The number of elements of the inner most circle (N1) is taken as 8 , for outermost circle (N3) as 12 , whereas for the middle circle (N2) as 10. The excitation amplitudes are allowed to vary in between 0 and 1 . Further, $\emptyset^{\circ}=0^{\circ}$ is assumed so that the radiation patterns of the CCAA of main lobe starts from $\emptyset^{0}=0^{\circ}$. In this experiment the algorithm parameters are set as follows:

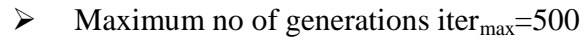

$>$ Initial no of seeds $=10$

$>$ Maximum no of seeds $\operatorname{Smax}=5$

$>$ Minimum no of seeds Smin=1

$>$ Maximum no of plants $=10$

$>$ Initial Standard deviation $\sigma_{\text {ini }}=0.05$

$>$ Final Standard deviation $\sigma_{\text {fin }}=0.000001$

$>$ Modulation index $\mathrm{n}=3$

Fig.3 shows the radiation pattern for a uniformly excited CCAA $\left(I_{m n}=1\right)$, it has a radiation pattern with $-9.26 \mathrm{~dB}$ side lobe level and a BWFN of $62^{\circ}$.

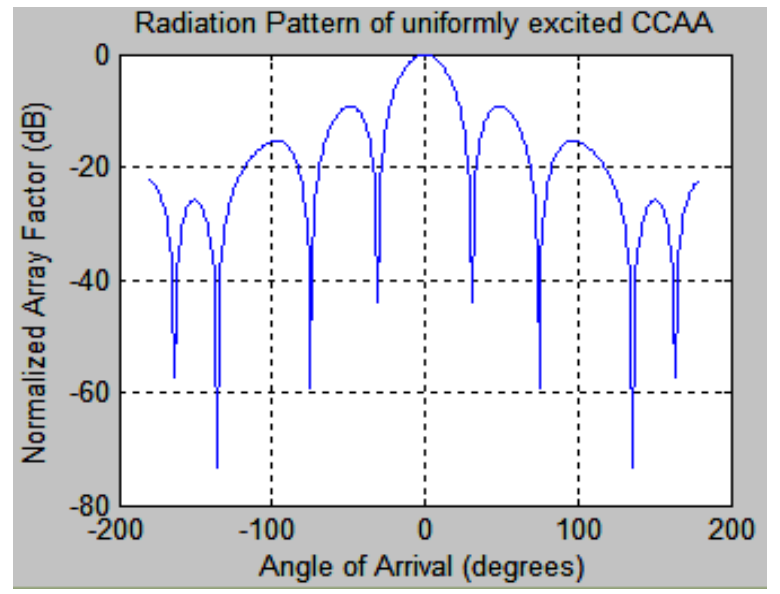

Fig 3: Radiation pattern of uniformly excited CCAA

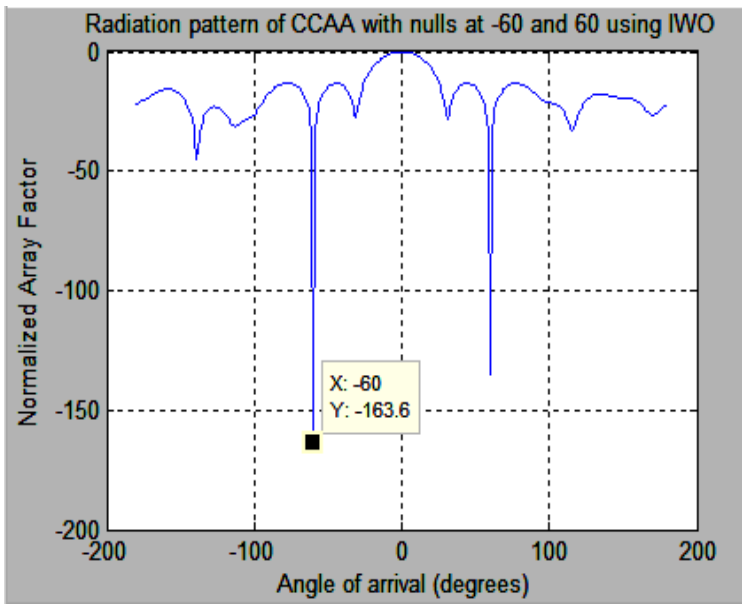

Fig 4: Radiation pattern of CCAA with nulls at $-60^{\circ} \& 60^{\circ}$

Fig. 4 shows radiation pattern of CCAA with nulls imposed at -60 and 60 degrees. It can be seen that nulls with depth of $163.6 \&-135.4 \mathrm{~dB}$ respectively were imposed at -60 and 60 degrees while SLL reduced to $-13.19 \mathrm{~dB}$ with FNBW unaltered. The Array Factor value prior to optimization at -60 and 60 degrees (in uniform excitation case) was $-11.99 \mathrm{~dB}$. 


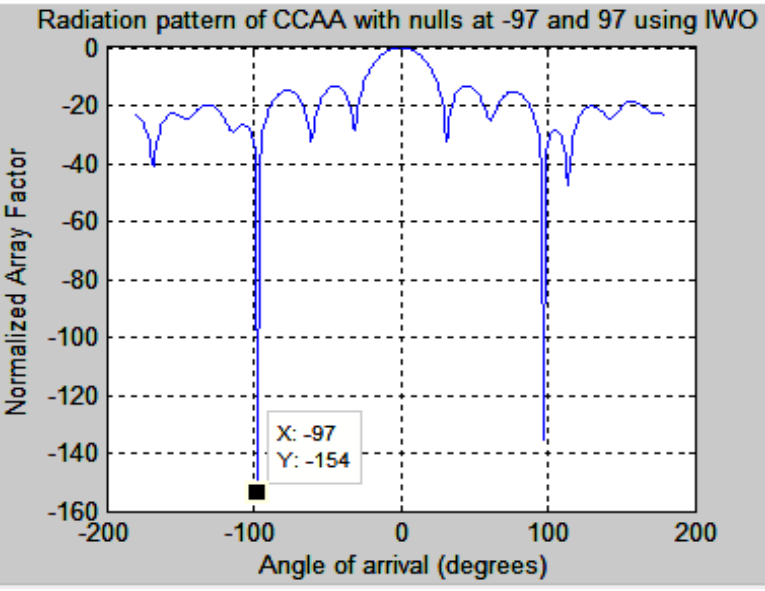

Fig 5: Radiation pattern of CCAA with nulls at $-97^{0} \& 97^{0}$

Fig.5 shows radiation pattern of CCAA with nulls imposed at -97 and 97 degrees where there are second side lobe peaks in the reference pattern (uniform excitation case). It can be seen that nulls with depth of $-154 \&-135.6 \mathrm{~dB}$ respectively were introduced while SLL reduced to $-13.14 \mathrm{~dB}$ with FNBW unaltered. The Array Factor value prior to optimization at -97 and 97 degrees (in uniform excitation case) was $-15.33 \mathrm{~dB}$.

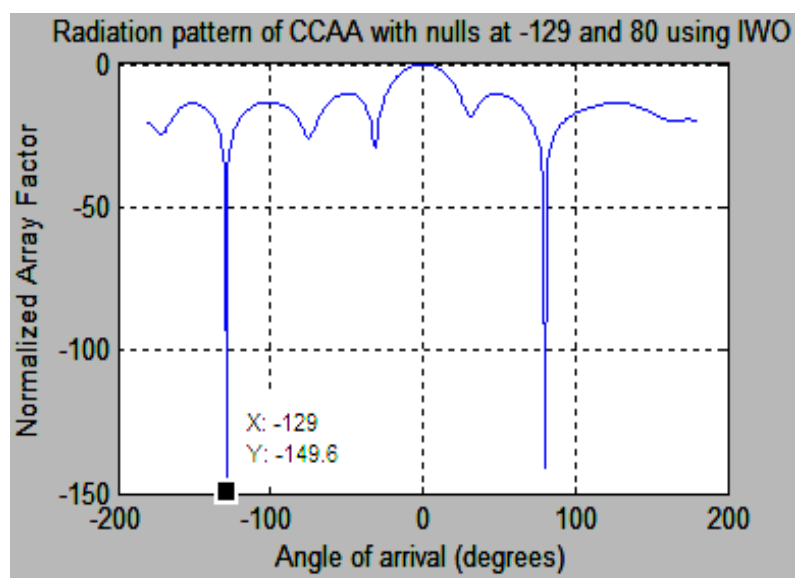

Fig 6: Radiation pattern of CCAA with nulls at $-129^{\circ} \&$ $\mathbf{8 0}^{0}$

Fig.6 shows radiation pattern of CCAA with nulls introduced at directions non-symmetric about the main lobe peak. It can be seen that nulls with depth of -149.6, -141.4 dB respectively were imposed at -129 and 80 degrees respectively while SLL reduced to $-10.13 \mathrm{~dB}$ with FNBW unaltered. The Array Factor values prior to optimization at -129 and 80 degrees (in uniform excitation case) were respectively -27.99 $\&-23.2 \mathrm{~dB}$.

Table-1 lists the excitation amplitudes for Uniform and non-uniformly excited cases.

Table 2. Lists the SLL, FNBW, Null depths for different cases and compares them with the uniform excitation case.
Table 1. Excitation Amplitudes of Uniform and NonUniform (Optimized) Cases OF CCAA

\begin{tabular}{|c|c|c|}
\hline Case & \multicolumn{2}{|c|}{ Excitation Current Amplitudes } \\
\hline Uniform & $\mathrm{Imn}=1 ; \mathrm{m}=$ & $\mathrm{n}=1, \ldots, \mathrm{Nm}$ \\
\hline $\begin{array}{c}\text { Nulls at } \\
-60 \text { and } \\
60 \\
\text { degrees }\end{array}$ & $\begin{array}{l}0.863189841606609 \\
0.015027135775206 \\
0.454039510137097 \\
0.002251280084882 \\
0.514055180073436 \\
0.167263176772073 \\
0.351031993637044 \\
0.667742576815755 \\
0.002199683344687 \\
0.065949938909426 \\
0.672160452277381 \\
0.430834477971242 \\
0.007101838771039 \\
0.353826274866413 \\
0.777568641847976\end{array}$ & $\begin{array}{c}0.131880054064926 \\
0.027862652520328 \\
0.004332873129706 \\
0.284692276644535 \\
\\
0.103521952856483 \\
0.054318132431273 \\
0.591960152103409 \\
0.060586036020286 \\
0.437152797190516 \\
\\
0.966201228270046 \\
0.731495975246870 \\
0.974977118585171 \\
0.983811165874774 \\
0.749264368799921 \\
0.871889732644568\end{array}$ \\
\hline $\begin{array}{c}\text { Nulls at } \\
-97 \& \\
97 \\
\text { degrees }\end{array}$ & $\begin{array}{l}0.922369091990832 \\
0.118998485313511 \\
0.945269020859707 \\
0.049667867530708 \\
0.404781975961038 \\
0.109027565799071 \\
0.257079219676442 \\
0.145161887080539 \\
0.056783463321405 \\
0.024159654346829 \\
0.665164163837642 \\
0.413061238107922 \\
0.229320692761072 \\
0.563570329012114 \\
0.840568993955446\end{array}$ & $\begin{array}{c}0.007310764750460 \\
0.052454427214364 \\
0.212960086171007 \\
0.103993274319817 \\
\\
0.448159081390768 \\
0.018154048017065 \\
0.111706541666976 \\
0.012369673922327 \\
0.534193952604411 \\
\\
0.921694292559652 \\
0.530842148353855 \\
0.914120427365701 \\
0.934930055145555 \\
0.794226589387215 \\
0.820625255046704\end{array}$ \\
\hline $\begin{array}{c}\text { Nulls at } \\
-129 \& \\
80 \\
\text { degrees }\end{array}$ & $\begin{array}{l}0.512918106672987 \\
0.485607190499808 \\
0.999428443635531 \\
0.072927375233130 \\
0.340461691179451 \\
0.927678988345303 \\
0.933010509118488 \\
0.171285700510188 \\
0.427810524796174 \\
0.843468066773909 \\
0.515383583385414 \\
0.663257122515318 \\
0.270714776615277 \\
0.340096050420651 \\
0.931043890286367\end{array}$ & $\begin{array}{c}0.071143416914631 \\
0.810449216458126 \\
0.446679604233458 \\
0.233379283587196 \\
\\
0.226433745227026 \\
0.022364806529045 \\
0.922091232111400 \\
0.111689968221311 \\
0.189351846500122 \\
\\
0.608551551698412 \\
0.507557114296393 \\
0.288620407355105 \\
0.992880437612003 \\
0.877544327759371 \\
0.224439656224466\end{array}$ \\
\hline
\end{tabular}


Table 2. SLL, Null Depths and FNBW of Uniform and Non-Uniform (Optimized) Cases of CCAA

\begin{tabular}{|c|c|c|c|}
\hline Case & $\begin{array}{c}\text { Sidelobe } \\
\text { Level (SLL) } \\
(\mathbf{d B})\end{array}$ & $\begin{array}{c}\text { FNBW } \\
\text { (degrees) }\end{array}$ & $\begin{array}{c}\text { Null depths } \\
\text { at intended } \\
\text { position (dB) }\end{array}$ \\
\hline Uniform & -9.266 & 62 & NA \\
\hline $\begin{array}{c}\text { Nulls at -60 \& } \\
60\end{array}$ & -13.19 & 62 & $\begin{array}{c}-163.6 \&- \\
135.4\end{array}$ \\
\hline $\begin{array}{c}\text { Nulls at -97 \& } \\
97\end{array}$ & -13.14 & 62 & $\begin{array}{c}-154.0 \&- \\
135.6\end{array}$ \\
\hline $\begin{array}{c}\text { Nulls at -129 } \\
\text { and 80 }\end{array}$ & -10.13 & 62 & $\begin{array}{c}-149.6 \&- \\
141.4\end{array}$ \\
\hline
\end{tabular}

The same problem was also implemented using RCGA in [4]. The Genetic algorithms are explained in [7-8]. The average values of the cost function for the 3-ring CCAA implemented using RCGA and IWO is given in table 3. It can be seen that the cost function obtained using IWO is approximately 10,000 times smaller than that of RCGA's. Thus IWO converges to a more optimum solution than RCGA. IWO also requires less number of CF calculations than RCGA. It is found that the number of $\mathrm{CF}$ calculations required to be performed during the optimization process using IWO is approximately $21.44 \%$ of that of RCGA's. The IWO is thus more accurate, efficient and even more time saving than RCGA.

Table 3. Average Cost Function Values of the 3-Ring CCAA Implemented Using RCGA and Iwo

\begin{tabular}{|c|c|}
\hline Algorithm & Cost Function Value \\
\hline RCGA & $7.1229806930026690 \mathrm{e}-08$ \\
\hline IWO & $8.1113502022232802 \mathrm{e}-12$ \\
\hline
\end{tabular}

\section{CONCLUSION}

The simulation results show that deeper nulls were introduced in the interference directions. Sidelobe levels were also reduced considerably while maintaining fixed FNBW. IWO algorithm is proved to be simpler, less resource consuming yet more robust and efficient algorithm than RCGA. IWO is also more efficient than PSO, ACO and Simulated Annealing.
The Future research could focus on other array geometries like rectangular arrays, volumetric arrays, Circular array or CCAA with center element etc. Research could be expanded to thinning of antenna arrays where optimization of number of elements of the arrays is required. In this area, IWO algorithm outplays other algorithms as IWO can use different seeds with varying number of parameters.

\section{REFERENCES}

[1] Kwai-Man Luk and Kai Fong Lee "Antennas in Wireless Communications" Proceedings of the IEEE , Vol. 100, No. 7, July 2012

[2] C. A. Ballanis, "Antenna theory analysis and design," $2^{\text {nd }}$ edition, John Willey and Son's Inc. • New York, 1997.

[3] Mandal,D.; Chandra,A.; "Side Lobe Reduction of a Concentric Circular Antenna Array using Genetic Algorithm", Serbian Journal of electrical engineering , vol.7, No.2, Nov. 2010, On pages 141-148.

[4] Thotakura T Ramakrishna Satish Raj and P Krishna Kanth Varma, "Real Coded GA (RCGA) for Control of Nulls and Sidelobes in a Concentric Circular Antenna Array (CCAA)" IJRECE Vol. 3 Issue 2 Apr-June 2015

[5] Shaya Karimkashi And Ahmed A. Kishk, "Invasive Weed Optimization And Its Features In Electromagnetics" IEEE Transactions On Antennas And Propagation, Vol. 58, No. 4, April 2010

[6] A. R. Mallahzadeh, "Application of The Invasive Weed Optimization Technique for Antenna Configurations", Progress In Electromagnetics Research, PIER 79, 137150,2008

[7] R. L. Haupt, and D. H. Werner, "Genetic Algorithms in Electromagnetics”, IEEE Press Wiley-Interscience, 2007.

[8] Randy L. Haupt, Sue Ellen Haupt, "Practical Genetic Algorithms", 2nd edition, Wiley- Interscience, John Wiley \& Sons, INC., Publication. 